Supporting Information

\title{
Atomistic Molecular Dynamics Simulations of Crude Oil/Brine Displacement in Calcite Mesopores
}

\author{
Mohammad Sedghi, Mohammad Piri, and Lamia Goual \\ Department of Petroleum Engineering, University of Wyoming, 1000 East University Avenue, \\ Laramie, Wyoming 82071, United States
}

* Corresponding author: msedghi@uwyo.edu 


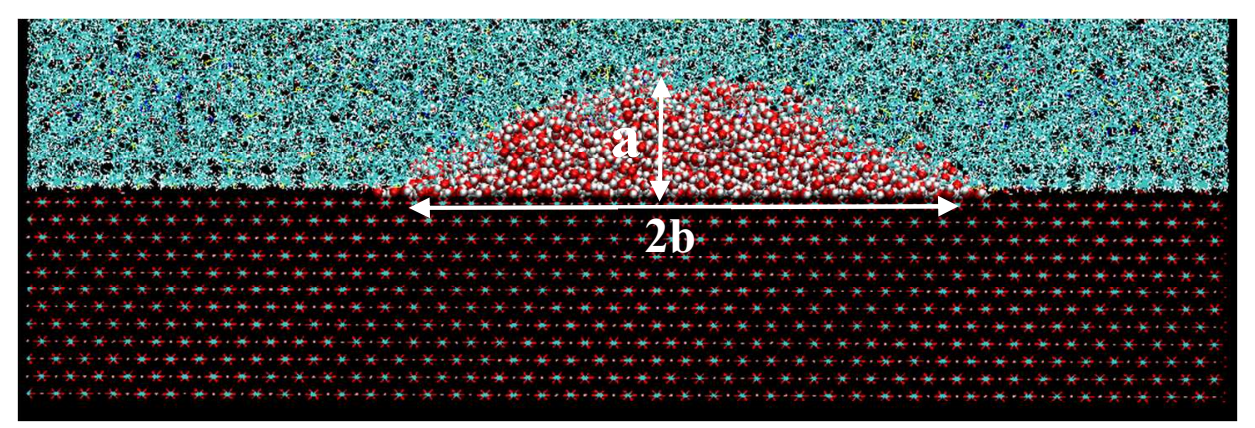

Figure S1. Snapshot of a water droplet (red and white particles) on calcite surface surrounded by oil molecules (cyan particles).

Table S1. Molecular structures of nonpolar organic components of the crude oils used in this study

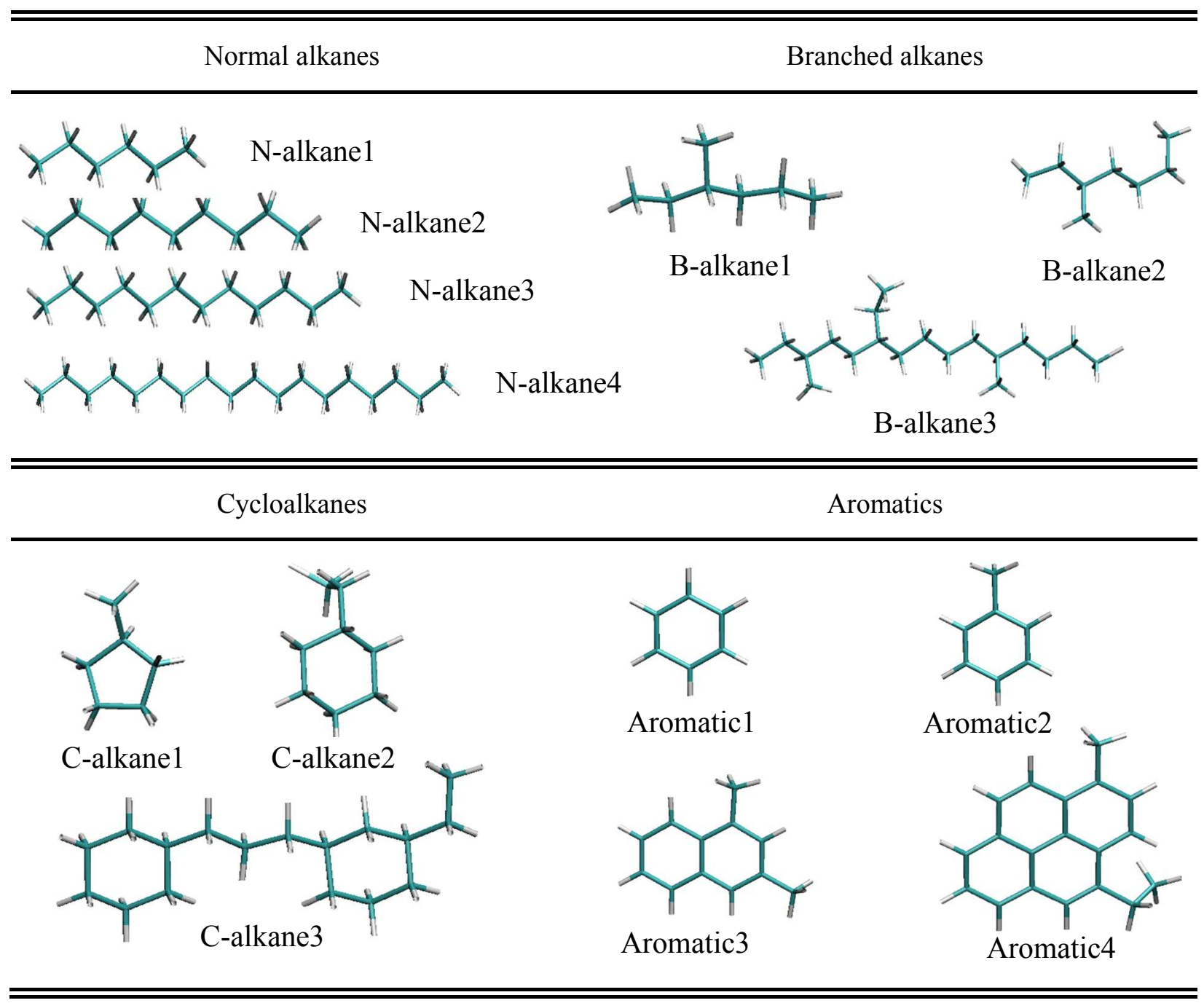


Table S2. Average molecular weight and weight percentage of the nonpolar oil fractions

\begin{tabular}{lcc}
\hline \hline & MW & Wt\% \\
\hline Normal alkanes & 148.7 & 25 \\
Branched alkanes & 154.0 & 25 \\
Cycloalkanes & 140.6 & 15 \\
Aromatics & 119.3 & 35 \\
\hline \hline
\end{tabular}


Table S3. Total number of water and oil molecules used for the IFT simulations (the names of nonpolar molecules are shown in Table S1)

\begin{tabular}{lcc}
\hline \hline & Oil-B - Water & Oil-A - Water \\
\hline Water & 6922 & 6922 \\
N-alkane1 & 64 & 128 \\
N-alkane2 & 42 & 84 \\
N-alkane3 & 20 & 40 \\
N-alkane4 & 43 & 86 \\
B-alkane1 & 61 & 122 \\
B-alkane2 & 54 & 108 \\
B-alkane3 & 48 & 96 \\
C-alkane1 & 37 & 74 \\
C-alkane2 & 38 & 76 \\
C-alkane3 & 33 & 66 \\
Aromatic1 & 101 & 202 \\
Aromatic2 & 86 & 172 \\
Aromatic3 & 79 & 158 \\
Aromatic4 & 60 & 58 \\
CRBZ & 60 & - \\
QUIN & 60 & - \\
INDO & 60 & - \\
BZAC & 60 & - \\
NPAC & 60 & - \\
NNAC & 60 & - \\
NNON & 60 & - \\
PRPH & 60 & - \\
NTHL & 60 & - \\
ZTHL & 60 & - \\
NNSL & 60 & - \\
\hline \hline
\end{tabular}




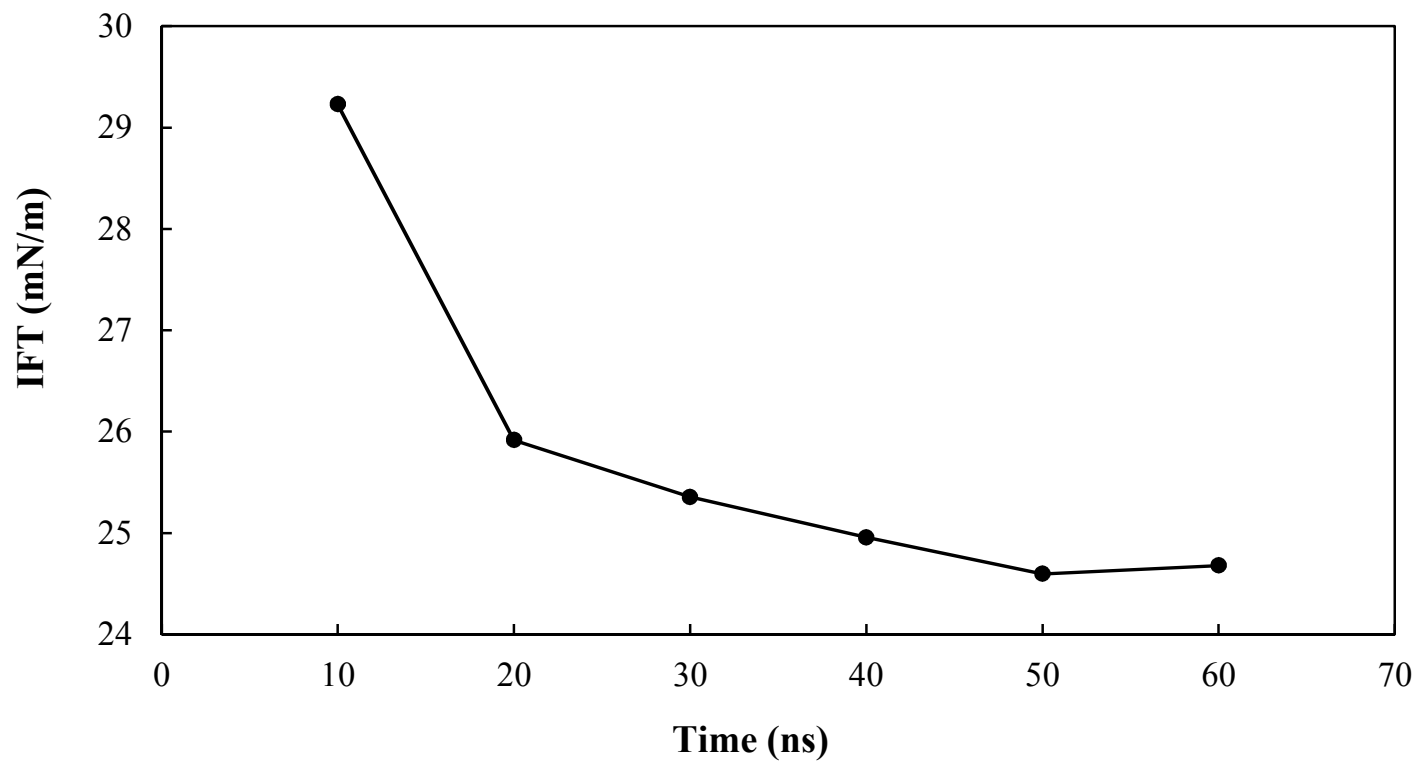

Figure S2. Change of IFT with time for oil B - water system at ambient conditions

Table S4. Composition of the brine used in the MD simulations

\begin{tabular}{lccc}
\hline \hline & $\mathbf{C a}^{2+}$ & $\mathbf{N a}^{+}$ & $\mathbf{C L}^{-}$ \\
\hline Concentration $[\mathrm{mg} / \mathrm{l}]$ & 15320 & 78950 & 141510 \\
\hline \hline
\end{tabular}

Table S5. Total Coulomb energy of water and brine at ambient and reservoir conditions

\begin{tabular}{lc}
\hline \hline Component & Coulomb energy, $\mathrm{kJ} / \mathrm{mol}$ \\
\hline Water at Amb cond. & $-3.86 \mathrm{E}+05$ \\
Water at Res cond. & $-3.39 \mathrm{E}+05$ \\
Brine & $-5.88 \mathrm{E}+05$ \\
\hline \hline
\end{tabular}


Table S6. Coulomb energies between water molecules and ions in brine

\begin{tabular}{lc}
\hline \hline Component & Coulomb energy, $\mathrm{kJ} / \mathrm{mol}$ \\
\hline Water-Water & $-1.76 \mathrm{E}+05$ \\
Water-Na $^{+}$ & $-1.33 \mathrm{E}+05$ \\
$W^{2+}-\mathrm{Ca}^{2+}$ & $-3.35 \mathrm{E}+04$ \\
$W^{2}-\mathrm{Cl}^{-}$ & $-1.51 \mathrm{E}+05$ \\
$\mathrm{Na}^{+}-\mathrm{Cl}^{-}$ & $-8.30 \mathrm{E}+04$ \\
$\mathrm{Ca}^{2+}-\mathrm{Cl}^{-}$ & $-4.74 \mathrm{E}+04$ \\
\hline \hline
\end{tabular}

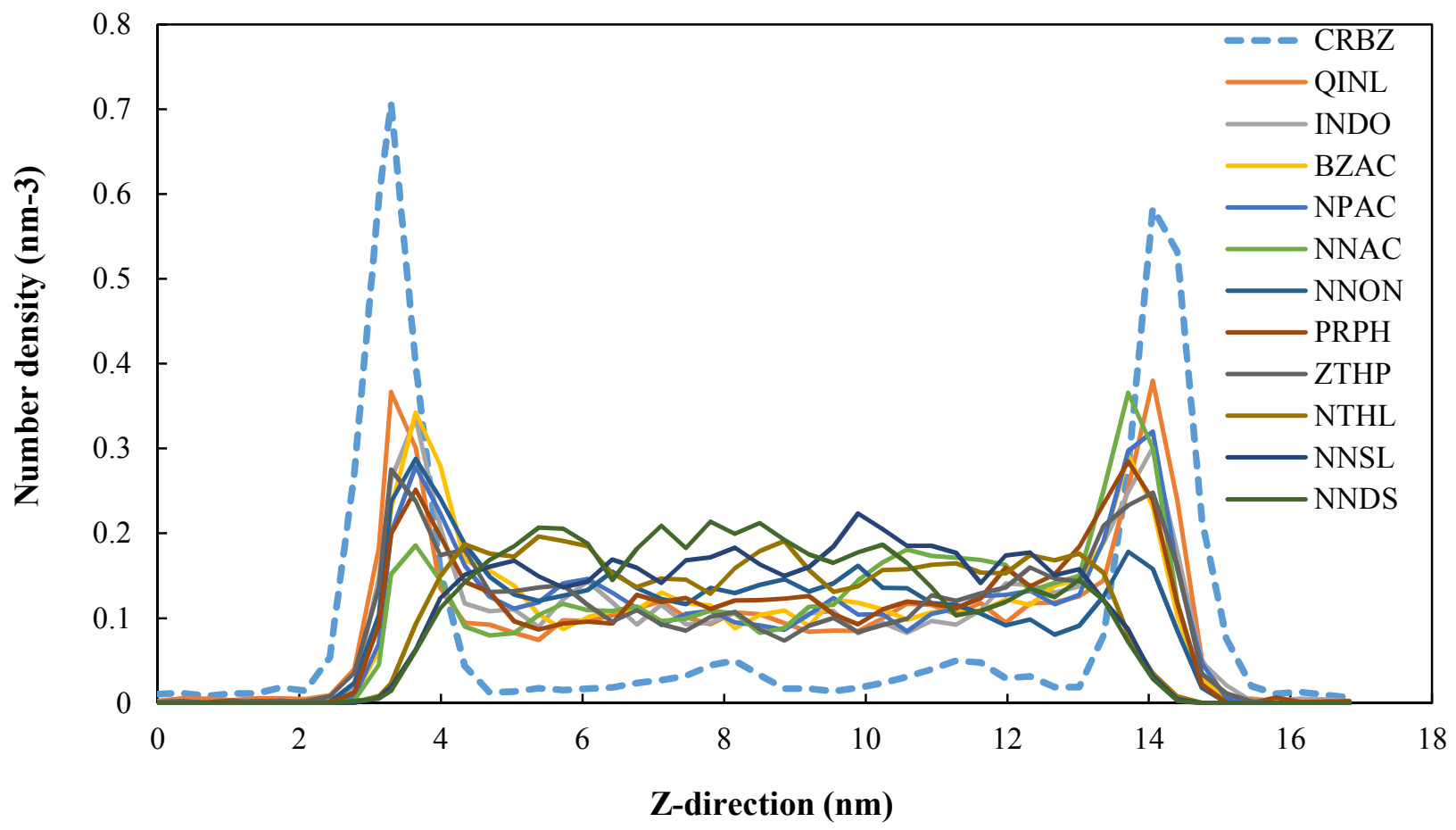

Figure S3. Density profiles of oil polar molecules in the $\mathrm{Z}$ direction during the simulation of oil B/water IFT at reservoir conditions (the abbreviation of the name of polar molecules is mentioned in Table 1 of the manuscript). 


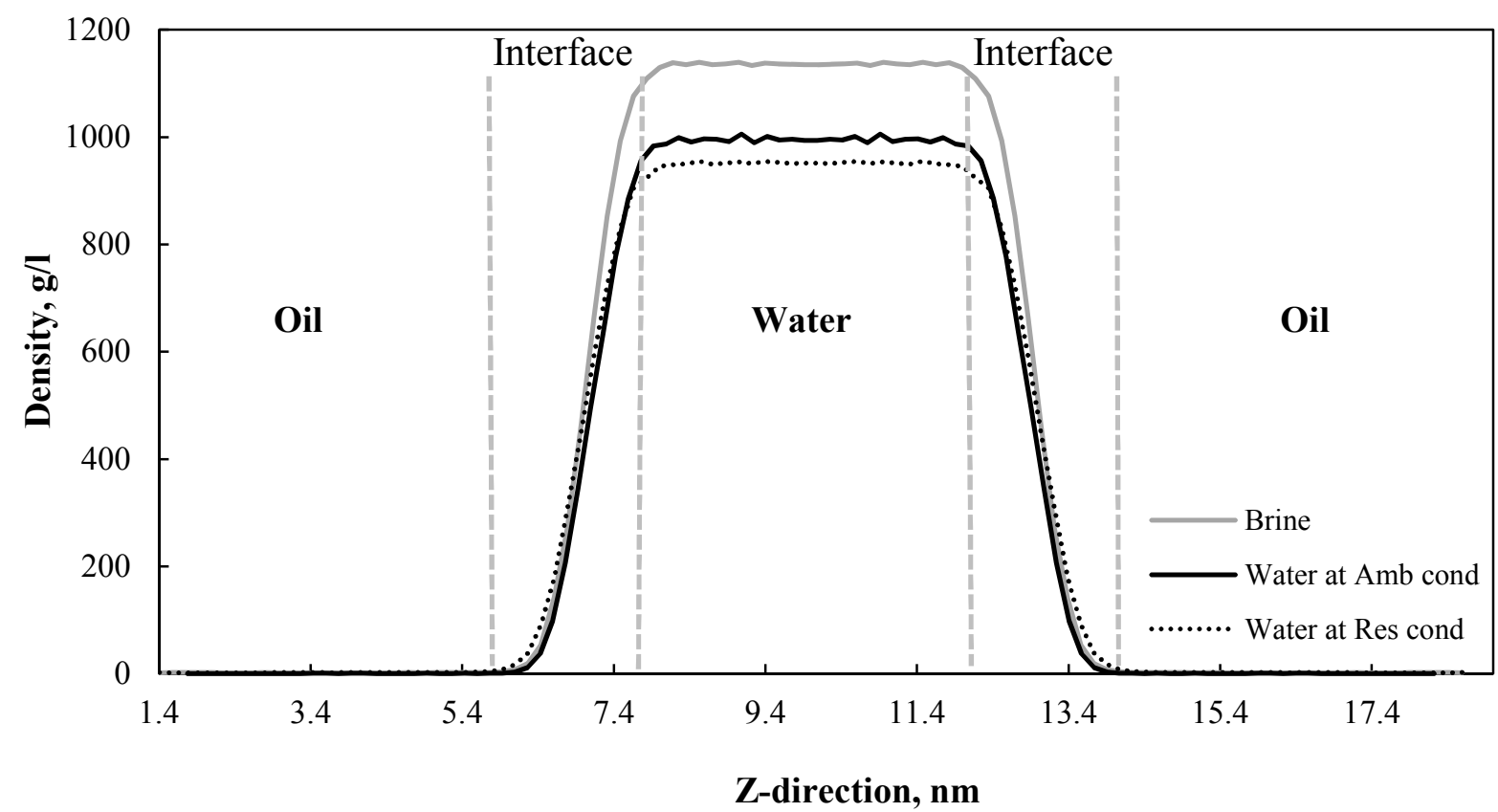

Figure S4. Density profiles of water and brine during the IFT simulations at ambient and reservoir conditions.

Table S7. Relative densities of oil polar molecules at the oil/brine interface at reservoir conditions

\begin{tabular}{lc}
\hline \hline Component & Relative density \\
\hline Carbazole & $\mathbf{1 7 . 6 9}$ \\
Quinoline & 2.05 \\
Indole & 1.76 \\
Benzoic acid & 1.98 \\
Naphthenic acid & 2.47 \\
Nonanoic acid & 2.03 \\
Nonanone & 1.81 \\
Propylphenol & 1.66 \\
Benzothiophene & 1.92 \\
Nonanethiol & 0.35 \\
Methyloctyl-Sulfide & 0.30 \\
Methyloctyl-Disulfide & 0.28 \\
\hline \hline
\end{tabular}


Table S8. Relative densities of oil polar molecules at the oil/water interface at ambient conditions

\begin{tabular}{lc}
\hline \hline Component & Relative density \\
\hline Carbazole & $\mathbf{5 . 9 4}$ \\
Quinoline & 2.18 \\
Indole & 2.24 \\
Benzoic acid & 2.19 \\
Naphthenic acid & 1.32 \\
Nonanoic acid & 1.49 \\
Nonanone & 2.55 \\
Propylphenol & 2.01 \\
Benzothiophene & 2.42 \\
Nonanethiol & 0.14 \\
Methyloctyl-Sulfide & 0.18 \\
Methyloctyl-Disulfide & 0.09 \\
\hline \hline
\end{tabular}



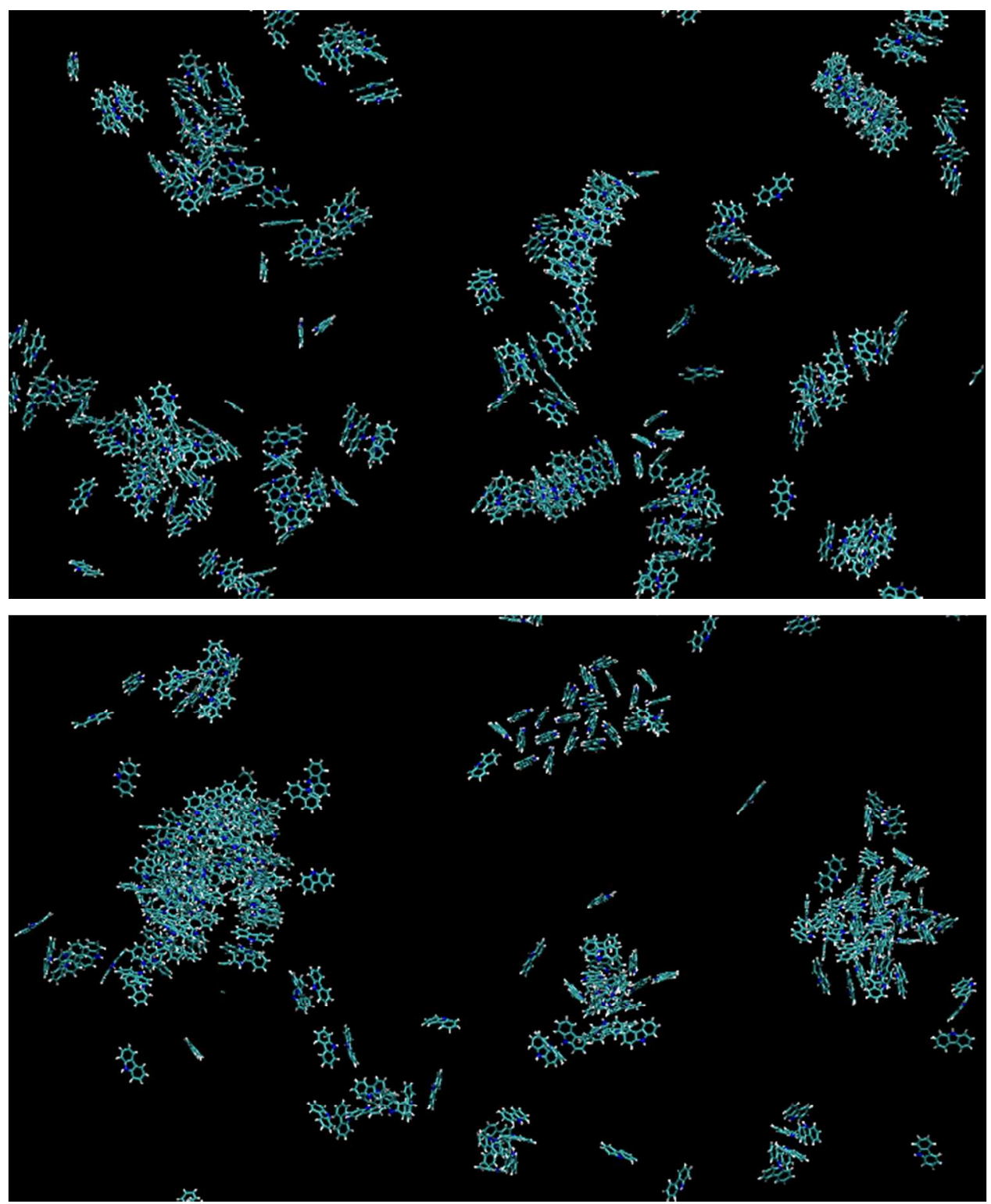

Figure S5. Snapshot of carbazole aggregates in oil B at ambient (top) and reservoir (bottom) conditions. 
Table S9. Buckingham parameters between $\mathrm{Ca}^{2+}$ and heteroatoms ( $\mathrm{O}$ and $\left.\mathrm{N}\right)$ found in the polar molecules of crude oil

\begin{tabular}{llllc}
\hline \hline & & $\mathrm{A}(\mathrm{eV})$ & $\rho(\mathrm{Ang})$ & $\mathrm{C}\left(\mathrm{eV} \mathrm{Ang}^{-6}\right)$ \\
\hline $\mathrm{Ca}^{2+}$ & $\mathrm{N}$ (Carbazole) & 1774.45 & 0.253 & 0.0 \\
$\mathrm{Ca}^{2+}$ & $\mathrm{N}$ (Indole) & 4173.54 & 0.253 & 0.0 \\
$\mathrm{Ca}^{2+}$ & $\mathrm{N}$ (Quinoline) & 3253.91 & 0.253 & 0.0 \\
$\mathrm{Ca}^{2+}$ & $\mathrm{O}(=\mathrm{C})$ (Naphthenic acid) & 1937.77 & 0.297 & 0.0 \\
$\mathrm{Ca}^{2+}$ & $\mathrm{O}(-\mathrm{H})$ (Naphthenic acid) & 2062.91 & 0.297 & 0.0 \\
$\mathrm{Ca}^{2+}$ & $\mathrm{O}(=\mathrm{C})$ (Nonanoic acid) & 1937.77 & 0.297 & 0.0 \\
$\mathrm{Ca}^{2+}$ & $\mathrm{O}(-\mathrm{H})$ (Nonanoic acid) & 2062.91 & 0.297 & 0.0 \\
$\mathrm{Ca}^{2+}$ & $\mathrm{O}(=\mathrm{C})$ (Benzoic acid) & 1708.02 & 0.297 & 0.0 \\
$\mathrm{Ca}^{2+}$ & $\mathrm{O}(-\mathrm{H})$ (Benzoic acid) & 1836.09 & 0.297 & 0.0 \\
$\mathrm{Ca}^{2+}$ & $\mathrm{O}$ (Nonanone) & 1758.51 & 0.297 & 0.0 \\
$\mathrm{Ca}^{2+}$ & $\mathrm{O}$ (Propylphenol) & 1886.59 & 0.297 & 0.0 \\
\hline \hline
\end{tabular}




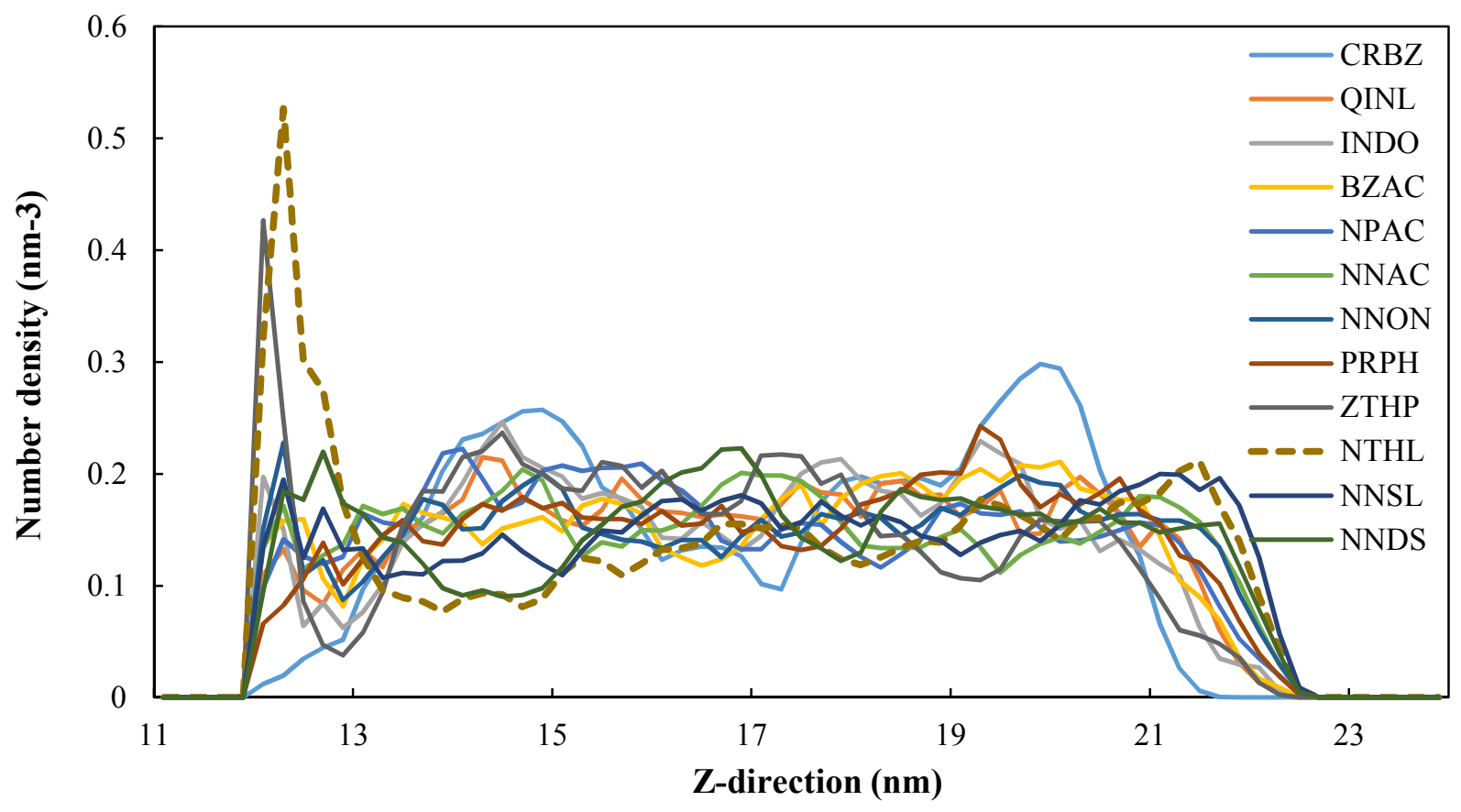

Figure S6. Density profiles of oil polar molecules on the surface of calcite (the abbreviation of the name of polar molecules is mentioned in Table 1 of the manuscript). 